\title{
Awareness Of Early Detection And Prevention Of Oral Cancer among Dentists - A Review.
}

\author{
Sabana A. Nazar; Dr. T.N.Uma maheswari, \\ Final year student, saveetha dental college and hospitals, saveetha university, 162, poonamalle high road, \\ Chennai 600077 India. \\ Associate professor Department of Oral Medicine and Radiology Saveetha dental college and hospital \\ .Saveetha university, 162. Poonamalle high road, Chennai-600 077 India.
}

\begin{abstract}
Assessment of knowledge of early diagnosis of oral cancer among dental population is needed for early treatment to ensure good prognosis. Knowledge about recent trends in diagnosis, treatment and preventive measures in oral cancer is utmost essential for improving the quality of life in oral cancer patients. Oral cancers are still diagnosed in advanced stages only and the main reason is because of lack of awareness among public and dentists. Early detection, which comprises screening of asymptomatic population and creating awareness of public regarding early signs and symptoms of oral cancer, increases the probability of cure. This article aims in review of knowledge of dentists in early diagnosis, evaluation of dentist's practice of anti-tobacco counseling and assessment of dentist knowledge on recent trends in diagnosis of cancer- risk potential in three different countries where oral pharyngeal cancer is more prevalent.
\end{abstract}

Key Words: Oral cancer, early detection, dentist awareness, Prevention.

\section{Introduction:}

Oral and pharyngeal cancer, grouped together, is the sixth most common cancer in the world with the annual estimated incidence is around 275,000 for oral. In high-risk countries such as Sri Lanka, India, Pakistan and Bangladesh, oral cancer is the most common cancer in men, and may contribute up to $25 \%$ of all new cases of cancer [1]. Tongue is the most common site for intraoral cancer among European and the US populations, amounting to $40-50 \%$ of oral cancers [2]. Approximately 34,360 cases of oral cancer and pharynx cancer are reported in the United States of America [3]. In United Kingdom there were 4660 new cases of oral and pharyngeal cancer diagnosed in 2003 and accounted for $1.6 \%$ of all new cancers [4]. Cancer lesions with immediate referral to a head and neck surgeon or cancer treatment center for biopsy and treatment of cancer is the best way to manage such cases and proper screening for evaluation of metastasis to or in some cases from other sites like breast, pancreas etc., is also mandatory [5]. Since most of the oral cancer arises from premalignant lesions and is usually asymptomatic, routine dental screening is essential for early diagnosis [6]. $43 \%$ of cancer death is due to tobacco, unhealthy diet, physical inactivity and infection. Tobacco and alcohol consumption have been estimated to account for about $90 \%$ of cancer in the oral cavity [7]. India is second most producer of tobacco with approximately 274.9 million tobacco users [8]. Almost $90 \%$ of cancer is squamous cell carcinoma [9]. Oral pharyngeal cancer commonly occurs in the age of 45, particularly those who smoke and drink alcohol. The recent increase in Oral pharyngeal cancer may be associated with Human papilloma virus, Hepatitis C virus, Hepatitis B virus. Prevention is critically important through early detection and tobacco use cessation [10]. Early detection of oral cancer is treatable [11]. Nearly overall providers agreed that early detection improves 5 year survival rates of Oral pharyngeal carcinoma [12]. Awareness of Oral cancer early detection, prevention and knowledge about recent trends among dentists is one of the best ways to manage oral cancer. Hence this review article aims in assessment of knowledge of dentists in early diagnosis, proper screening and early referral to oncologists in three different countries namely United States of America, United Kingdom and India as oral cancer rate is considerably high in these countries.

\section{United States of America:}

In the USA, oral pharyngeal cancer ranks as the 4th leading cancer site in black men, and 6th common site in white men. A total of 1892 general dentists in private practice attended 64 standardized continuing education courses, throughout the ten public health districts of the USA. Questionnaires were obtained at baseline and at 6 months along with matched control groups. 1892 general dentists participated at baseline and $988(52.2 \%)$ completed the questionnaire 6 months later. Of the 1015 controls completing the questionnaire at baseline, 678 also completed it at 6 months. Greater than $95 \%$ of all groups recognized the importance of oral pharyngeal cancer detection. $82.6 \%$ of participants understood the steps comprising Oral pharyngeal cancer screening. A significant improvement to $92.7 \%$ at the 6 months follow up and greater than $90 \%$ of both attendees and controls stated that they performed oral pharyngeal cancer examination, and younger dentists were 
more likely to perform Oral pharyngeal cancer screenings than their older colleagues. Examination of neck palpation increases from $60 \%$ at baseline to $69.1 \%$ in 6 months follow up. Oral pharyngeal cancer screenings at recall visits increased average of $2 \%$ over initial visit examination for all age groups across all groups, only approximately $70 \%$ indicated that they informed patients when they were performing an Oral pharyngeal cancer examination. Continued education courses had positive influence though reported that adjunctive diagnostics were not used commonly by the dentists who attended the course at baseline even after six months [10].

\section{India:}

In literature search in assessing the awareness of early detection, prevention of oral pharyngeal cancer among Indian dental population revealed that there are very few surveys conducted in India. Recently (2012) a survey was conducted in the Bangalore city to assess the knowledge, attitude, screening practices of general dentists concerning oral cancer in Bangalore city. A descriptive cross sectional study was conducted using a 24 item self administered questionnaire involving 240 dental practitioners. The results of the survey revealed that less than $37 \%$ of dentists were involved in anti-tobacco counseling. This survey also proved that alcohol was the major risk factor for that alcohol as reported by $99 \%$ of the dentists. 59\% of dentists also reported that fourth and fifth decades as high risk age of oral cancer. This survey declared strongly the need for educational interventions for practitioners and dental students, though $98 \%$ of dentists referred the suspected oral cancer patients to oncologist [13].

\section{United Kingdom:}

A questionnaire survey was carried out in the year 1991. This questionnaire has 13 test items which was educated at continuing education courses and then distributed to dentist's subscribers of the British Dental Journal. The questionnaire concentrated mainly on the recent attempts taken by the dentists in updating their knowledge on oral cancer. The questionnaire also included the practical approaches in screening for oral mucosal diseases and follow-up after oral screening. Questionnaire asked to the dental patients regarding the major risk factors for oral cancer to assess the public awareness. The questionnaire was circulated to 15,836 dentists and the response rate is very less and account to be $16 \%$. In 2519 responses, the results of the questionnaire survey revealed that $84 \%$ are involved in screening of the oral mucosa routinely. $74 \%$ reported referral of screen detected cases to oncology centers for further management. $4 \%$ adopted a wait and see policy. The results also revealed that most of the dentists did not inquired risk habits related to oral cancer and even if when few dentists claimed to make such enquiries, only $30 \%$ provided health education advice to such patients. Patient's compliance was reported as $71 \%$. This survey concludes that there is a need for patient counseling to be provided by the dental practitioners [9].

\section{Discussion:}

In this review article of assessment of dentist awareness of knowledge and screening practice of oral cancer included totally 4651 dentists (USA 1892, India 240, UK 2519)[10][13][9]. This review article reveals the fact that inspite of availability of various recent invasive and non invasive diagnostic modalities; most of the dentists are not trained in using the specialized diagnostic tools during their routine dental practice of examining potentially malignant disorders. This is evident because the cure rates and 5 year survival is reported as $60 \%$ among the oral pharyngeal cancer patients [10]. Professional education is equally important as public education as it ensures most effective control of oral pharyngeal carcinoma. Results of the Indian survey proved that $24 \%$ of dentists subject the suspected oral cancer lesions to biopsy [13]. Early detection awareness among dental population in these three countries is calculated and found to be $2.48 \%$ and prevention awareness is $1.31 \%$. In India only $12 \%$ of the dentists referred patients to specialist clinics for further evaluation [13], whereas in UK dental population $74 \%$ dentists had referred patients to concerned specialists [9].

\section{Authors suggestions:}

This review article emphasizes the demand for extensive surveys to be conducted in different regions of the countries where oral pharyngeal cancer is highly prevalent. The results of this survey will be helpful in reflecting the hidden causes for negligence of early detection of oral pharyngeal carcinoma in various geographical areas within the countries. Routine cancer screening programs among all dental patients and applications of minimally invasive diagnostic tools in potentially malignant lesions should be emphasized in all dental hospitals. Continuing education courses, workshops, and specialized training for cancer screening camps should be emphasized in all dental institutions. Advanced non invasive diagnostic modalities to assess cancer risk potential both in healthy and malignant disorders like sialodiagnosis of tumour markers, VELscope, Autofluorescence spectro-photometry, etc is the current need for early detection of cancer and prevention of further progression of malignancy in cancer risk patients. All these measures once practiced will definitely decrease the incidence of cancer death and increase the percentage rate of survival in oral pharyngeal cancer patients. 


\section{References:}

[1] Ferlay J, Pisani P, Parkin DM. GLOBOCAN 2002. Cancer incidence, mortality and prevalence worldwide. IARC Cancer Base (2002 estimates). 2004; Lyon: IARC Press

[2] Saman Warnakulasuriya, Global epidemiology of oral and oropharyngeal cancer .Oral Oncology. (2009); 45: 309-316.

[3]. Surveillance epidemiology and end results (SEER). SEER Cancer statistics review 1975-2004. National Cancer Institute. Available from: http://seer.cancer.gov/statfacts/html/oralcav (accessed 03.03.08).

[4]. Cancer Research Campaign. Cancer Stats. Oral cancer - UK. UK: CRC; April 2005.

[5]. Little JW, Cancer awareness and dentistry. Gen Dent 2000 Jul-Aug; 48(4):462-5.

[6]. M. McGurk \& S. E. Scott, The reality of identifying early oral cancer in the general dental practice. British Dental Journal 208, (2010); $347-351$.

[7] Stewart BW, kleihurs world cancer report Lyon. WHO International agency for research on cancer 2003.

[8] Oral cancer awareness of general public in Gorakhpur city, India, Asian pacific Journal of cancer prevention. 13 (10): $5195-5199$.

[9]. Dentist and oral cancer prevention in UK opinion, attitude and practices to screening for mucosal lesion and to counseling patients on tobacco and alcohol use: baseline data from 1991.oral diseases 1991: Jan 5(1); 10-4.

[10]. Oral and pharyngeal cancer control and early detection. J cancer educ. (2010); 25/: 279-281)

[11]. Prevention and early detection and pharyngeal cancer in veterans Oral Surg Oral med Oral path Oral Radiol Endo 2006 ; 102 :( 1758-64).

[12]. Adequacy of training in oral cancer prevention and screening as self assessed by physician, nurse practitioner and dental health professionals. Oral Surg Oral Med Oral path Oral Radio Endo 2006:102 (758-64).

[13]. Knowledge, attitude and screening practical of General dentist concerning oral cancer in Bangalore city. Indian journal of cancer $2012 ; 49: 33-8$ 